\title{
Modelling of the hydrodynamic conditions throughout liquid system treatment by alternating impulses of pressure
}

\author{
Iryna Dubovkina1, Borys Davydenko ${ }^{1}$, Veronica Rikhter ${ }^{2}$
}

\section{1 - Institute of Engineering Thermophysics of National Academy of Sciences of Ukraine, Kyiv, Ukraine 2 - Technical University of Wien, Wien, Austria}

\section{Keywords:}

Water

Pressure

Hydrodynamic

Impulse

Alternating

\section{Article history:}

Received 12.08.2018

Received in revised

form 23.12.2018

Accepted 31.05.2019

\section{Corresponding} author:

Iryna Dubovkina

E-mail:

dubovkinai@

ukr.net

DOI: $10.24263 / 2304-$

974X-2019-8-2-13

\section{Abstract}

Introduction. The purpose of this scientific work is to research the influence of the application of alternating impulses of pressure throughout treatment of liquid solutions by the numerical modelling.

Materials and methods. The volume parametric imitation and visualization, modelling methods, math modelling methods were used for the description of the conditions at the working parts of the rotary pulsed apparatus during the liquid treatment. Experimental investigations of liquid samples were carried out with using potentiometry laboratory measurement procedure.

Results and discussion. In this paper, it is established that the local values of pressure in the sector of input of water and water systems and the output of the water systems from the gaps vary: at the external surface of the internal rotor from -50 to $+300 \mathrm{kPa}$; at the external stator surface from -150 to $100 \mathrm{kPa}$; at the inner stator surface from +40 to $-120 \mathrm{kPa}$; at the inner surface of the external rotor from +100 to $-100 \mathrm{kPa}$. During the last values cavitation and adiabatic boiling can be present.

Throughout the water and water solutions treatment and during the processes of mixing liquid associated components in the conditions of hydrodynamic fluctuation as alternating impulses of pressure characterized the changing of pressure: $\Delta \mathrm{P}=350 \mathrm{kPa}$ near an outside surface of an interior spinning rotor; $\Delta \mathrm{P}=250 \mathrm{kPa}$ near an outside stator surface; $\Delta \mathrm{P}=160 \mathrm{kPa}$ near an interior stator surface; $\Delta \mathrm{P}=200 \mathrm{kPa}$ near an interior surface of an outside spinning rotor.

By the consequences of the computation was established that the principal values of speeds of shift of a stream emerge in RPA with coaxial clearances between first rotors, stator and second rotor $100 \mathrm{mkm}$.

Conclusions. Throughout the calculations was found the values of pressure changing in working chamber of rotary-pulsating apparatus between coaxial cylinders «rotor-stator-rotor» system. These give us the possibility to receive the liquids with necessary and required physical and chemical properties and parameters by the no reagent influence and to control this processes. 


\section{Introduction}

At the present time a great variety of technological processes of foodstuff manufacture have to reduce energy consumption and to intensify industrial modes.

One of the way to solve this problem is to employ innovative nonstandard energy and resource saving and inexpensive equipment and new optimized modes of treatment.

There are many methods and processes of water treatment to obtain water and water solutions with necessary physical and chemical parameters and properties which require for the manufacturing.

They are including: acoustic treatment, the electromagnetic pulse effect of the lowfrequency field, cavitations processing, emitting treatment (ultraviolet, ionizing, infrared), hydrodynamic effects [1-3].

The method of discrete-pulsed input of energy can power structural transformations in difficult liquid systems on micro- and nanolevel and gives possibility to initiate physical and chemical transformations in these complex systems.

The main effects of the discrete-pulsed input of energy are effects which connected with increase of velocity of association of a continuous phase, power of pressure of shift, cavitations, the effect of explosive boiling, collective effects in assembly of vials, crossness of an interphase surface in gas-liquid bubbly medium, action of hydrodynamic oscillations, alternating impulses of pressure.

A great number of mass engineering processes such as: mixing, homogenization, crushing, dispersion, activating, emulsification, etc are exhausted in rotary pulsed apparatus of different type.

The main effects of the discrete-pulsed input of energy are realising in these types of apparatus.

To intensify mixing of associated mediums it is necessary to use rotary pulsed apparatus. In such equipment the treatment of liquid mediums occurs with the passage throughout thin annular clearance [4].

This clearance is formed by the surfaces of coaxial cylinders which turn around common axis (rotors) and inexperienced cylinder (stator).

There are many slits on the rotors and stator. During the treatment slits can coincide on the rotors and stators. Significant pressures of the shift and microcirculation streams emerge as a result of the treatment of liquid heterogeneous solutions in working chamber and parts of the apparatus.

Self-oscillation mode can occur throughout the treatment of liquid mediums at rotary pulsed apparatus. The oscillations of velocities and pressures in mediums which processed are characterizing self-oscillation mode [PI UA № 117998, A method for preparation of disinfectant].

High energy transitional hydro-mechanical influence on liquid mediums and solutions in rotary pulsed apparatus conducts to transformation of structural formation and in the same time there is no intensive destruction of macromolecules and associates.

To optimize the process of hydrodynamic treatment it is necessary to define the level of power influence on the liquid mediums and solutions for indispensable transformations which can provide predictable physical and chemical parameters.

The main factors and driving forces that cause them are the stresses of the pressures of shift of a stream, the speeds of shift of a stream, alternating impulses of pressure.

The main features of the technological mediums processing are:

- the mechanical influence on the particles of a heterogeneous mediums consisting of shock and abrasive loads during contact with the working parts of the rotary pulsed apparatus; 
- the hydrodynamic effect, which manifests itself in significant tangential stresses in the fluid, developed turbulence, pressure pulsations and fluid flow speeds;

- the hydroacoustic influence on the liquid system is carried out by the microscale pressure pulsations, intense cavitation, shock waves and nonlinear acoustic effects $[5,6]$.

For a complete description of the mixing process and the treatment modes, the first necessary condition is the establishment of qualitative and quantitative coherency between the properties and the physical and chemical parameters of the initial components.

It is significant to establish the coherency between technological modes of carrying out the mixing process and the treatment modes, the design and constructor features of the working apparatus and the properties and physical and chemical parameters of the resulting mixtures.

It is important to use the parameters that should directly reflect the nature of the energy effect on the liquid medium. It is also necessary to use parameters reflecting the nature of the energy action on the particles of substances.

Accordingly, the essential conditions are:

- the establishing of connection between the constructional parameters of the rotary pulsed apparatus with the energy effect, which is achieved at the same time;

- the determination of the dependence of the main technological parameters of the treatment process on the intensity of the energy impact and influence.

On the basis of the numerical experiment and analytical calculation, the main constructional parameters of the apparatus were found which influence the intensification of the processes of mixing liquid components and treatment of the liquid technological mediums.

The purpose of this scientific work is to research the influence of the application of alternating impulses of pressure throughout treatment of liquid solutions by the numerical modelling.

\section{Materials and methods}

\section{Materials}

Liquid solutions and systems: water, alcohol, water-alcoholic solutions and mixtures in a wide range of concentration (percentage of alcohol in mixtures was varied from 5 to $90 \%$ ), distilled aqua, hydroponic [7], hydroponic with fertilizers [8] were used for experiments.

\section{Experimental installation}

The object of this scientific research work was rotary pulsed apparatus in which liquid solutions treat by alternating impulses of pressure, the speeds of shift of a stream, pressures of shift of a stream. Rotary pulsed apparatus was the cylindrical type with the working parts «rotor-stator-rotor» [9].

\section{Methods}

General scientific methods and special methods, such as potentiometry were used for the analyzing of the results of research work [10].

The volume parametric imitation and visualization, modelling methods, math modelling methods were used for the description of the conditions at the working parts of the rotary pulsed apparatus during the liquid treatment. 
Experimental investigations of liquid samples were carried out with using potentiometry laboratory measurement procedure [11].

For the description of physical and chemical parameters of liquid samples of water and solutions which obtained throughout the experimental investigations, chemical methods described in singular literature are used [12].

For the reception related data, liquid samples of water and solutions were analyzed not less than three times with the following statistical processing [13].

\section{Results and discussion}

\section{Numerical simulation model}

The numerical simulation and visualization was employed for hydrodynamic computation of the treatment and mixing processes in working volume (chamber) of the rotary pulsed apparatus (RPA).

For the choice of numerical model of fluid flow in the «rotor-stator-rotor» system of the RPA, it was suggested that the current in the prevailing case is two-dimensional.

The heterogeneous stream was considered to be a homogeneous medium with the effective thermophysical properties.

To consider the dynamics difficulty, the horizontal section of the working parts of the RPA, perpendicular to the axis of the «rotor-stator-rotor» system, was selected. This section has slits on the surfaces of the first rotor, stator and second rotor. Also this section consists of the left and right parts of the rotors.

In addition that takes place geometrical and physical periodicity of the processes in the geometrical similar sections. That's why border conditions at left and right sides of the section are considered as a periodic.

In accordance with [14], the dimensionless system of fluid dynamics equations was chosen. For calculations, the dimensionless system of fluid dynamics was recorded in polar coordinates [15].

The midpoint of this section locates on the axis of the frame «rotor-stator-rotor» system.

Dimensionless equation system (1)-(5):

$$
\begin{aligned}
& \frac{1}{R} \frac{\partial(R V)}{\partial R}+\frac{\partial \Omega}{\partial \theta}=0 \\
& \frac{\partial V}{\partial H}+\frac{1}{R} \frac{\partial\left(R V^{2}\right)}{\partial R}+\frac{\partial(V \Omega)}{\partial \theta}-\Omega^{2} R=-\frac{\partial P}{\partial R}+\frac{2}{\operatorname{Re} R} \frac{\partial}{\partial R}\left(R \beta \frac{\partial V}{\partial R}\right)- \\
& -\frac{2}{\operatorname{Re} R} \beta\left(\frac{\partial \Omega}{\partial \theta}+\frac{V}{R}\right)+\frac{1}{\operatorname{Re} R^{2}} \frac{\partial}{\partial \theta}\left(\beta \frac{\partial V}{\partial \theta}\right)+\frac{1}{\operatorname{Re}} \frac{\partial}{\partial \theta}\left(\beta \frac{\partial \Omega}{\partial R}\right) ; \\
& \frac{\partial \Omega}{\partial H}+\frac{1}{R^{2}} \frac{\partial\left(R^{2} \Omega V\right)}{\partial R}+\frac{\partial \Omega^{2}}{\partial \theta}+\frac{\Omega V}{R}=\frac{1}{R^{2}} \frac{\partial}{\partial \theta}\left[-P+\frac{2}{\operatorname{Re}} \beta\left(\frac{\partial \Omega}{\partial \theta}+\frac{V}{R}\right)\right]+ \\
& +\frac{1}{\operatorname{Re} R^{3}} \frac{\partial}{\partial R}\left[R \beta\left(R^{2} \frac{\partial \Omega}{\partial R}+\frac{\partial V}{\partial \theta}\right)\right]
\end{aligned}
$$




$$
\begin{gathered}
\frac{\partial \vartheta}{\partial H}+\frac{1}{R} \frac{\partial(R \vartheta V)}{\partial R}+\frac{\partial \vartheta \Omega}{\partial \theta}=\frac{1}{\operatorname{RePr}}\left[\frac{1}{R} \frac{\partial}{\partial R}\left(\Lambda R \frac{\partial \vartheta}{\partial R}\right)+\frac{1}{R^{2}} \frac{\partial}{\partial \theta}\left(\Lambda \frac{\partial \vartheta}{\partial \theta}\right)\right]+\beta S^{2} \\
S=\left[2\left(\frac{\partial V}{\partial R}\right)^{2}+2\left(\frac{\partial \Omega}{\partial \theta}+\frac{V}{R}\right)^{2}+\left(\frac{1}{R} \frac{\partial V}{\partial \theta}+R \frac{\partial \Omega}{\partial R}\right)^{2}\right]^{0,5}
\end{gathered}
$$

The adduction to the dimensionless form of the equation system made by the changing:

$$
\begin{gathered}
V=\frac{v_{r}}{\omega_{0} r_{0}} ; \Omega=\frac{\omega}{\omega_{0}} ; R=\frac{r}{r_{0}} ; H=\tau \omega_{0} ; \\
P=\frac{\left(p-p_{0}\right)}{\left(\rho \omega_{0} 2 r_{0}^{2}\right)} ; \quad \vartheta=\frac{\left(T-T_{0}\right) \rho C p}{\left(\omega_{0} \mu_{0}\right)} \\
\operatorname{Re}=\frac{\rho \omega_{0} \times r_{0}^{2}}{\mu_{0}} ; \quad \operatorname{Pr}=\frac{c_{p} \mu_{0}}{\lambda_{0}} ;
\end{gathered}
$$

$p_{\mathrm{o}}$ - the pressure of the medium behind the working chamber of the rotary pulsed apparatus; $T_{o}$ - the temperature of the medium behind the working chamber of the rotary pulsed apparatus;

$\omega=\frac{\vartheta}{r}$ - angular velocity;

$\omega_{0}$ - angular velocity of the rotor spinning;

$r_{0}$ - radius of the internal surface of the internal stator;

$\beta=\frac{\mu_{e f}}{\mu_{0}} ; \Lambda=\frac{\lambda_{e f}}{\lambda_{0}} ;$

$\mu_{e f}-$ effective values of the viscosity coefficient;

$\lambda_{e f}-$ effective values of the coefficient of thermal conductivity;

$\mu_{0},-$ conditional scale of the viscosity coefficient

$\lambda_{0}-$ conditional scale of the coefficient of thermal conductivity.

Since rotary-pulsating apparatuses belong to periodic devices, it is necessary to pay attention to the fact that there is a geometrical periodicity of the design of the «rotor-statorrotor» system of working chamber.

In this connection, the frequency of changes in the dynamic characteristics of the fluid flow through the working area of the RPA is observed. When the RPA mode is set, the movement of the fluid is repeated after rotating the rotors at a periodic angle $\Delta \theta$.

To solve this assigned problem, a certain segment was selected that periodically repeats the angle $\Delta \theta$. 
The segment contained a fragment of the «rotor-stator-rotor» system with a slits on the surfaces of the rotors and stator also clearance between these working elements of the system.

The pressure difference between the input and the output liquid solutions at the working volume (chamber) before and after treatment is $\Delta \mathrm{P}=50 \mathrm{kPa}$.

Geometric sizes of working elements were set according to constructional and design dimensions of RPA.

The speed of rotation of the rotor was given $n=48 \mathrm{sec}^{-1}$. In the calculation it was considered that the flow regime of the liquid in the RPA is turbulent, so for this case RNG $\mathrm{k}-\varepsilon$ model of turbulence was chosen [16].

The system of equations was reduced to dimensionless form.

By the volume three-dimensional parametric imitation visualization modelling processes, mathematical and numerical modelling was found that the value of the linear speeds of a stream should be within $20 \mathrm{~m} / \mathrm{s}$ for the first rotor and $25 \mathrm{~m} / \mathrm{s}$, for the second rotor.

The investigation of the structure of the stream, and the vortical nature of the fluid movement, was performed by numerical simulation of the flow dynamics based on the Navier-Stokes equations.

The main factors of the flow structure is determined by the fields of pressures, speeds, accelerations [17].

The changes of the pressure in different points of the working volume (chamber) have complicated pulsating character.

For the numerical simulation were selected different positions of the «rotor-statorrotor» system of working chamber.

It was selected for calculations the next points:

- on the external surface of the internal rotor,

- on the external stator surface,

- on the inner stator surface,

- $\quad$ on the inner surface of the external rotor.

The subsequently positions in working volume (chamber) between coaxial cylinders of the «rotor-stator-rotor» system were selected:

- open slit;

- half closed slit;

- $\quad$ half open slit;

- closed slit.

\section{Investigation of alternating impulses of pressure}

The profile of pressure fields in working volume between coaxial cylinders of the «rotor-stator-rotor» system during the ope $\vartheta \mathrm{n}$ slit are shown at the Figure 1.

It was established that speeds of shift of a stream should be equal to $2,0 \cdot 10^{5} \mathrm{~s}^{-1}$ for the first rotor and $2,5 \cdot 10^{5} \mathrm{~s}^{-1}$ for the second rotor.

Such values of the speeds of shift of a stream make available to intensive mixing and particle movement in continuous phase - water.

The value of pressure of shift of a stream must be $220 \mathrm{~Pa}$ for the first rotor and $230 \mathrm{~Pa}$, for the second rotor.

The visualization are showed the pressure fields in the first moment of time during the input of liquid components or solutions into a working chamber through the slits on the first rotor surface, stator surface and second rotor surface and among clearances between first rotor, than stator and second rotor at the certain segment. 

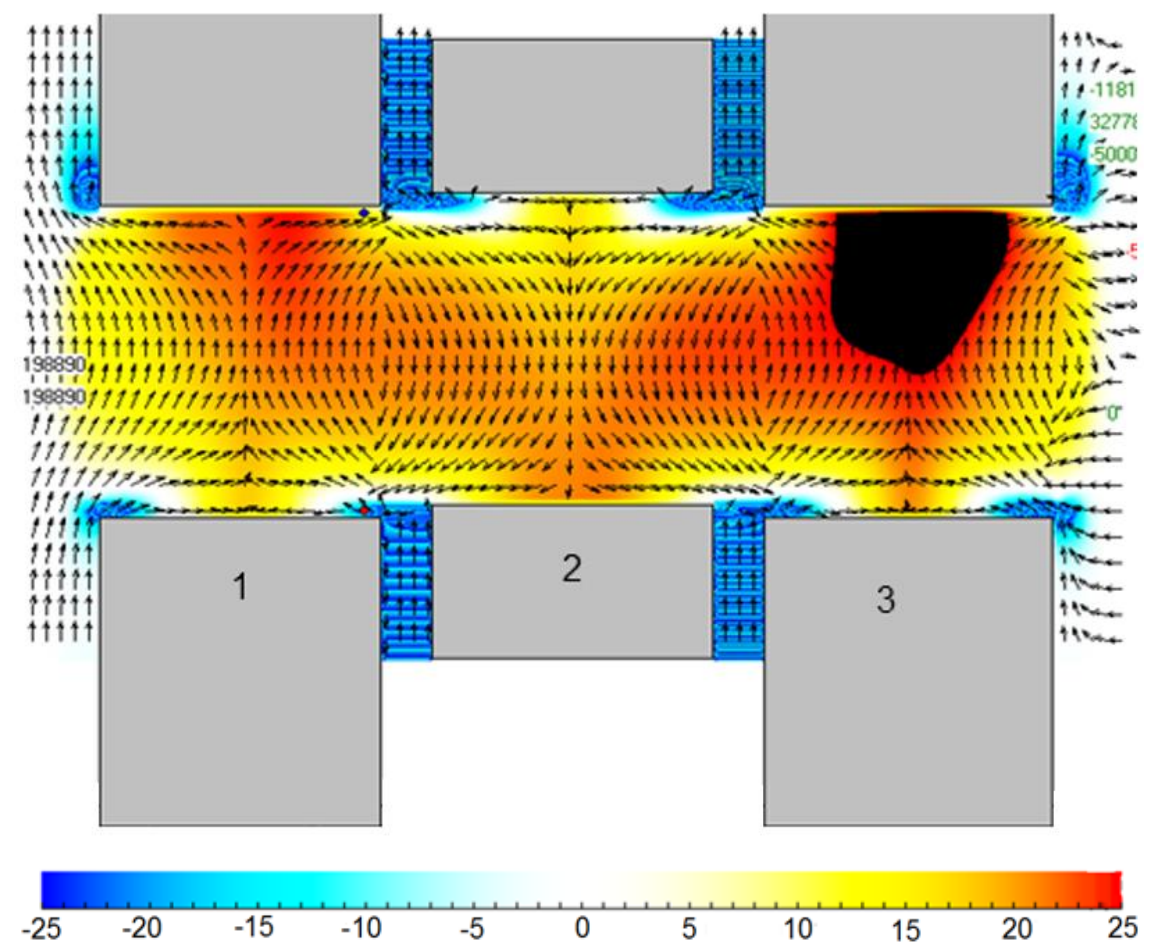

Figure 1. The profile of pressure fields in working volume (chamber) between coaxial cylinders (open slit): 1 - first rotor; 2 - stator; 3 - second rotor

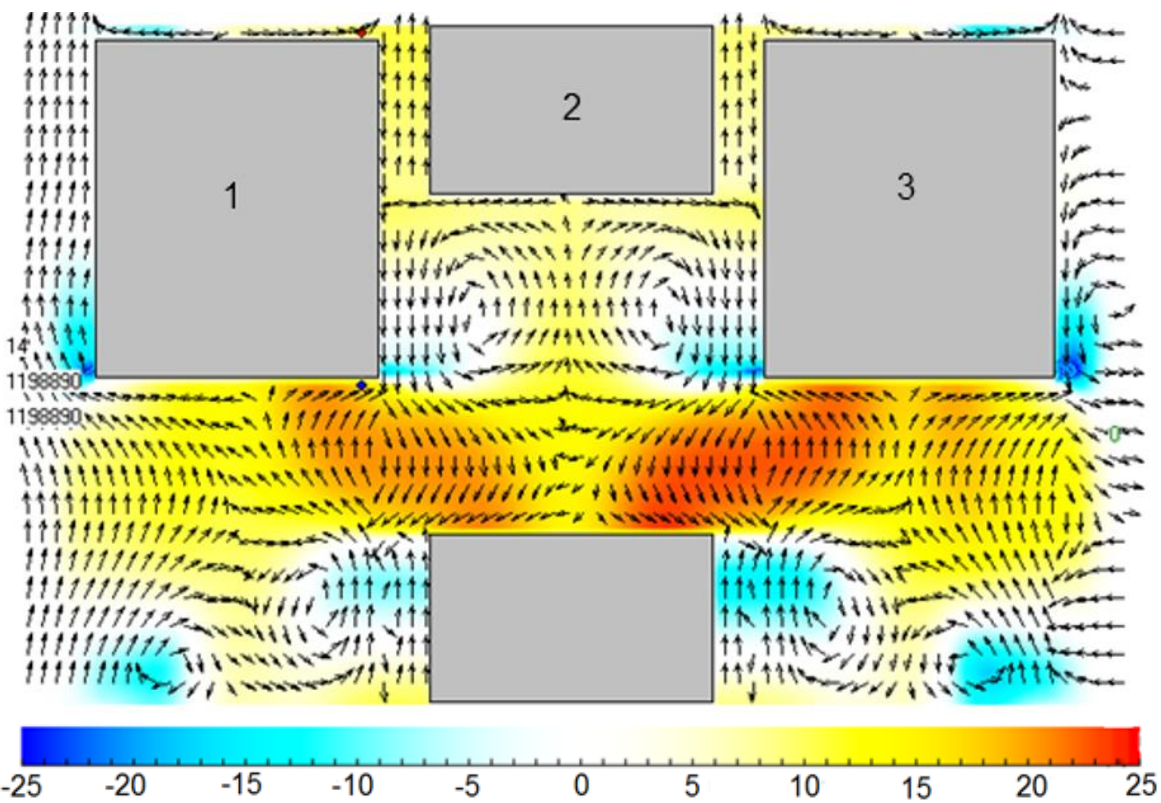

Figure 2. The profile of pressure fields in working chamber between coaxial cylinders (half open slit): 1 - first rotor; 2 - stator; 3 - second rotor 
Based on the results of the analysis of the calculations obtained, it was found that the reducing of the clearance between the external and internal rotors and the stator leads to an increase in the depth of the negative impulse of pressure.

Such impulses of pressure contribute to the intensification of the instantaneous mixing process, with the exception of the contact of the components before entering directly into the working area of the RPA. It is important factor for mixing of the specific chemical substances which react instantly.

The mass transfer intensification, in turn, affects the speed of the process of hydration, structuring and association of the polar liquids as alcohols and water.

The local values of pressure in the sector of input of water and water systems and the output of the water systems from the gaps vary:

- at the external surface of the internal rotor from -50 to $+300 \mathrm{kPa}$;

- at the external stator surface from -150 to $100 \mathrm{kPa}$;

- at the inner stator surface from +40 to $-120 \mathrm{kPa}$;

- at the inner surface of the external rotor from +100 to $-100 \mathrm{kPa}$.

During the last values cavitation and adiabatic boiling can be present [18].

Throughout the water and water solutions treatment and during the processes of mixing liquid associated components in the conditions of hydrodynamic fluctuation as alternating impulses of pressure characterized the changing of pressure:

- $\Delta \mathrm{P}=350 \mathrm{kPa}$ near an outside surface of an interior spinning rotor;

$-\Delta \mathrm{P}=250 \mathrm{kPa}$ near an outside stator surface;

- $\Delta \mathrm{P}=160 \mathrm{kPa}$ near an interior stator surface;

- $\Delta \mathrm{P}=200 \mathrm{kPa}$ near an interior surface of an outside spinning rotor.

\section{Investigation of Speed of shift of a stream and pressure of shift of a stream}

Important technical parameter such as speed of shift of a stream (Figure 5) was recognized for treatment of liquid solutions with the appliance of the alternating impulses of pressure.

By the consequences of the computation was established that the principal values of speeds of shift of a stream emerge in RPA with coaxial clearances between first rotors, stator and second rotor $100 \mathrm{mkm}$.

These demanding hydrodynamic conditions give the possibility to treat water system and water solutions [19] with the initialization of the formation of structure and intermolecular interacting such as forming three-dimensional framework from the hydrogen bonds. At a molecular scale, molecular dynamic simulation is an appropriate tool for analyzing these static and dynamic properties; as it provides a direct path from microscopic to macroscopic properties [20].

The nature and velocity of many physical and chemical processes which take place in such associated systems transforms.

Besides, the activity of the water depends from the transformations and hydrogen bonds which can form between molecules.

The changing of physical and chemical properties and parameters of pure water and water solutions has been established during the treatment with appliance of hydrodynamic fluctuation [21]. 


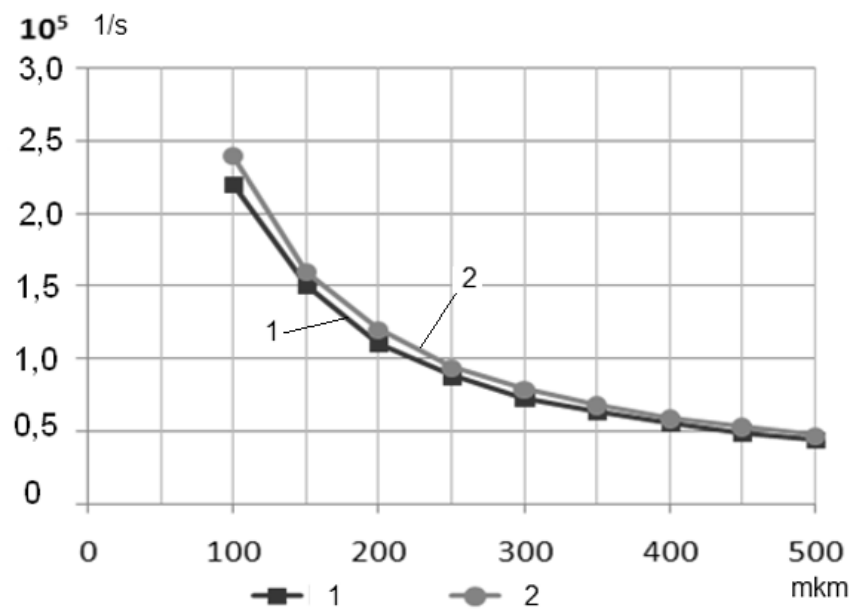

Figure 5. Profile of changes speeds of shift of a stream from the clearances between coaxial cylinders: 1 - first rotor; 2 - second rotor

By the results of the calculation was established that the largest values of pressure of shift of a stream emerge in RPA with coaxial clearances between rotors and stator $100 \mathrm{mkm}$ Figure 6.

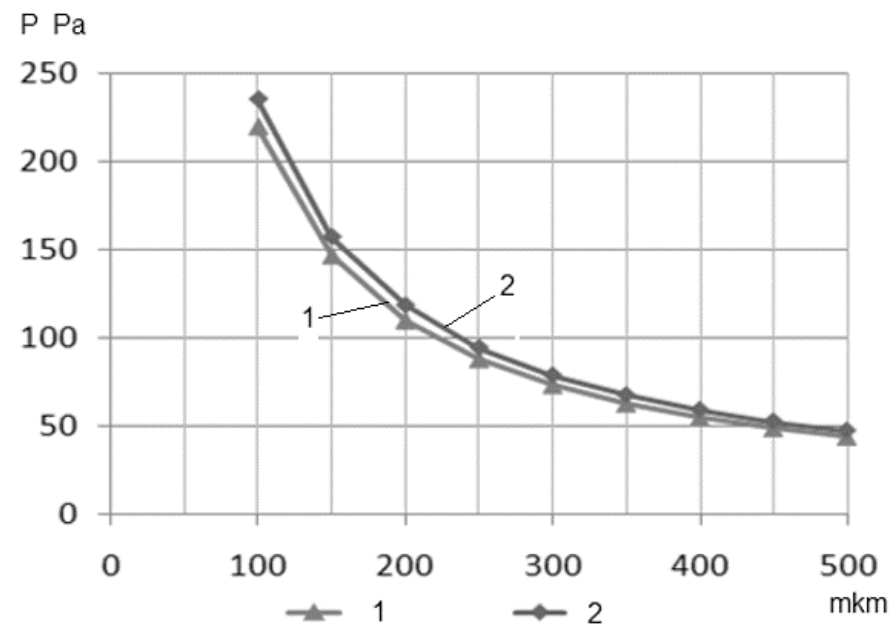

Figure 6. Profile of changes of pressure of shift of a stream from the clearances between coaxial cylinders: 1 - first rotor; 2 -second rotor

It was established that appliance of the alternating impulses of pressure for water solutions treatment give possibility to decrease redox potential on $20-70 \%$, to increase the potential of hydrogen on $13-17 \%$, to decrease mass of the dissolved oxygen on $50-55 \%$. [22] 


\section{Conclusions}

The application of alternating impulses of pressure throughout treatment of liquid solutions can influence on the physical and chemical parameters of the liquid systems by the hydrodynamic power.

Throughout the calculations was found the values of pressure changing in working chamber of rotary-pulsating apparatus between coaxial cylinders «rotor-stator-rotor» system. These give us the possibility to receive the liquids with necessary and required physical and chemical properties and parameters by the no reagent influence and to control this processes.

A detailed analysis of experimental data showed that the use of alternating impulses of pressure for treatment liquid solutions can be appropriate for processing in food engineering.

\section{References}

1. Doosti M.R., Kargar R., Sayadi M.H. (2012), Water treatment using ultrasonic assistance: A review, Proceedings of the International Academy of Ecology and Environmental Sciences, 2(2), pp. 96-110.

2. Philippe Vallee, Jacques Lafait, Laurent Legrand, Pascale Mentre, Marie Odile Monod, Yolene Thomas (2005), Effects of pulsed low frequency electromagnetic fields on water characterized by light scattering techniques: role of bubbles, Langmuir 2005, 21, 6, pp. 2293-2299.

3. Matevž Dular, Tjaša Griessler Bulc, Ion Gutiérrez Aguirre, Ester Heath, Tina Kosjek (2016), Use of hydrodynamic cavitation in (waste) water treatment, Ultrasonics Sonochemistry, 29, pp. 577-588.

4. Ning Zhang, Minguan Yang, Bo Gao, Zhong Li, Dan Ni (2016) Investigation of rotorstator interaction and flow unsteadiness in a low specific speed centrifugal pump, Strojniški vestnik, 62(1), pp. 21-31.

5. Yuequn Tao, Jun Cai, Xiulan Huai, Bin Liu, Zhixiong Guo (2016), Application of Hydrodynamic Cavitation to Wastewater Treatment, Chemical Engineering \& Technology, 39(8), pp. 1363-1376.

6. Slimane Merouani, Oualid Hamdaoui, Yacine Rezgui, Miloud Guemini (2015), Modeling of ultrasonic cavitation as an advanced technique for water treatment, Desalination and Water Treatment, 56(6), pp. 1465-1475.

7. Valeriy Myronchuk, Iryna Dubovkina (2017), Innovative method of water treatment in hydroponic system / University of Ruse “Angel Kanchev", Proceedings Of University Of Ruse, 56(10.2), p. 75-79.

8. Iryna Dubovkina, Oleksandr Ustinov (2018), Uninterrupted mode of water treatment for growing crops / Scientific Papers of the University of Ruse "Angel Kanchev", Proceedings Of University Of Ruse, 57(10.3), p. 50-55

9. Shurchkova J., Dubovkina I. (2015), Research parameters of the water-ethanol mixture obtained under conditions of alternating impulses of pressure, Bulletin of NTU "KhPI". Series: New solutions in modern technologies, 46(1155), pp. 171-176.

10. Singh V.P., Shalini Yadav, Ram Narayan Yadava (2018), Water quality management: select proceedings of ICWEES-2016, Water science and technology library, 79, Singapore, Springer.

11. Patrick J. Sullivan, Franklin J. Agardy, James J. J. Clark (2005), The environmental science of drinking water, Burlington, Elsevier, Butterworth-Heinemann. 
12. Laboratory manuals of American Water Works Association (2014), Simplified procedures for water examination, Denver, Colorado.

13. Kenneth I. Ozomwna (2007), Recent Advances in Analitycal Electrochemistry, Transword Research Network, Available at:

14. Network.http://www.ressign.com/UserBookDetail.aspx ?bkid=605\&catid=160

15. Basok B.I,. Davydenko B.V. (2016), Some features of hydrodynamic, heattransfer and the processes of dispersion in working volumes of cylindrical rotary pulsed apparatus, Micro and nanolevel processes in technologies DPIE, Kyiv, pp. 62-84.

16. Kleinstreuer C. (2018), Modern fluid dynamics, CRC Press, Boca Raton.

17. Yeon S Chang, Chapel Hill, Alberto Scotti USA (2004), Modeling unsteady turbulent flows over ripples: Reynolds-averaged Navier-Stokes equations (RANS) versus largeeddy simulation (LES), Journal of Geophysical Research: Oceans, 109(9), pp. 30-38.

18. Nakorchevskii I., Basok B.I., Ryzhkova T.S.(2002), Hydrodynamics of rotorypulsatory apparatuses, J. Engineering Physics and Thermodynamics, 75(2), pp. 338351.

19. Li Y., Zhang X., Guo D., Wang X., Li Y. (2018), Numerical analysis and verification of flow characteristics of rotor cavity of spiral rotary lobe pump, Nongye Gongcheng Xuebao, Transactions of the Chinese Society of Agricultural Engineering, 34(10), pp. 62-67.

20. Martin Petkovšek (2014), Rotation generator of hydrodynamic cavitation, Yearbook, pp. 74-77

21. Abdalla Obeidat; Rakan Al-Salman; Hind Abu-Ghazleh (2018), The validity of the potential model in predicting the structural, dynamical, thermodynamic properties of the unary and binary mixture of water-alcohol: Ethanol-water case, AIP Advances, 8(7), pp. $1-18$

22. Dubovkina I.O. (2015), Features of carrying out of process of mixing of water and spirit in the conditions of sign-variable impulses of pressure, Technology audit and production reserves, 6/1(26), pp. 42-45

23. Dubovkina Iryna (2017), Change of physical and chemical parameters of the liquid binary systems by alternating impulses of pressure, Ukrainian Food Journal, 6(1), pp. 142-154. 